\title{
Acceleration by Parallel Computations of Solving High-Order Time-Accurate Difference Schemes for Singularly Perturbed Convection-Diffusion Problems ${ }^{\star}$
}

\author{
Pieter W. Hemker ${ }^{1}$, Grigorii I. Shishkin ${ }^{2}$, and Lidia P. Shishkina ${ }^{2}$ \\ 1 CWI \\ Amsterdam, The Netherlands \\ P.W. Hemker@cwi.nl \\ 2 Institute of Mathematics and Mechanics, Ural Branch of RAS, \\ Ekaterinburg 620219, Russia \\ \{Grigorii, Lida\}@shishkin.ural.ru
}

\begin{abstract}
For singularly perturbed convection-diffusion problems with the perturbation parameter $\varepsilon$ multiplying the highest derivatives, we construct a scheme based on the defect correction method and its parallel variant that converge $\varepsilon$-uniformly with second-order accuracy in the time variable. We also give the conditions under which the parallel computation accelerates the solution process with preserving the higher-order accuracy of the original schemes.
\end{abstract}

\section{Introduction}

For several singularly perturbed boundary value problems, $\varepsilon$-uniformly convergent finite difference schemes have been constructed and analyzed (see, e.g., [1][5]). The time-accuracy of such schemes for nonstationary problems usually do not exceed first order. The use of a defect correction technique allows us to construct $\varepsilon$-uniform numerical methods with a higher order of accuracy in time (see e.g., [6,7]). Parallelization of the numerical method based on decomposition of the problem makes it possible to solve the discrete problem on a computer with several processors that may accelerate the computational process. However, this parallel process introduces additional errors in the numerical solutions. If the numerical method is accurate in time with order more than one, then the errors introduced by the domain decomposition (DD) can essentially exceed the discretization errors. Therefore, it is necessary to construct the parallel method such that the computation time is essentially less, and the accuracy is not lower than those for the corresponding nonparallel method.

\footnotetext{
* This research was supported in part by the Netherlands Organization for Scientific Research NWO, dossiernr. 047.008.007, and by the Russian Foundation for Basic Research under grant N 98-01-00362.
} 
In the case of singularly perturbed problems $\varepsilon$-uniform parallel schemes based on the defect correction principle were studied in [8]. Parallel methods that allowed us to accelerate the numerical solution of the boundary value problems for parabolic reaction-diffusion equations on an interval were developed in $[9,8]$.

In the present paper we consider the Dirichlet problem for a singularly perturbed convection-diffusion equation on a rectangle in that case when characteristics of the reduced equation are parallel to the sides of the rectangle. In this case regular and parabolic layers appear for $\varepsilon \rightarrow 0$. To solve the problem, we construct an $\varepsilon$-uniform scheme based on the defect correction method and its parallel variant convergent ( $\varepsilon$-uniformly) with second-order accuracy in time. We also write out the conditions under which the parallel computation accelerates the solution process without losing the accuracy of the original schemes. The technique for analysis of difference schemes is similar to that given in [8].

\section{Problem Formulation}

On the domain $G=D \times(0, T], D=(0,1) \times(0,1)$, with boundary $S=\bar{G} \backslash G$, we consider the Dirichlet problem for the singularly perturbed parabolic equation

$$
\begin{aligned}
L u(x, t) & \equiv\left\{\varepsilon^{2} \sum_{s=1,2} a_{s}(x, t) \frac{\partial^{2}}{\partial x_{s}^{2}}+b_{1}(x, t) \frac{\partial}{\partial x_{1}}-c(x, t)-p(x, t) \frac{\partial}{\partial t}\right\} u(x, t)= \\
& =f(x, t), \quad(x, t) \in G, \quad \\
u(x, t) & =\varphi(x, t), \quad(x, t) \in S .
\end{aligned}
$$

Here $a_{s}(x, t), b_{1}(x, t), c(x, t), p(x, t), f(x, t),(x, t) \in \bar{G}$, and $\varphi(x, t),(x, t) \in$ $S$ are sufficiently smooth and bounded functions, moreover, $a_{s}(x, t) \geq a_{0}>$ $0, b_{1}(x, t) \geq b_{0}>0, p(x, t) \geq p_{0}>0, c(x, t) \geq 0,(x, t) \in \bar{G} ; \quad \varepsilon \in(0,1]$.

Let $S=S^{L} \cup S_{0}, S_{0}=\bar{S}_{0}$. We distinguish four faces in the lateral boundary $S^{L}: S^{L}=\cup_{j=1}^{4} S_{j}, S_{j}=\Gamma_{j} \times(0, T]$, where $\Gamma_{1}, \Gamma_{2}, \Gamma_{3}$ and $\Gamma_{4}$ denote the left, bottom, right and top sides of the rectangle $D$ respectively.

When the perturbation parameter $\varepsilon$ tends to zero, regular and parabolic layers appear respectively in the neighborhood of the boundaries $S_{1}$ and $S_{2}, S_{3}$.

\section{Special Finite Difference Scheme}

On $\bar{G}$ we construct the piecewise uniform grid (see, e,g, $[10,3]$ )

$$
\bar{G}_{h}=\bar{D}_{h} \times \bar{\omega}_{0}, \quad \bar{D}_{h}=\bar{\omega}_{1} \times \bar{\omega}_{2} .
$$

Here $\bar{\omega}_{0}$ is a uniform mesh on $[0, T]$ with step-size $\tau=T / N_{0}, \bar{\omega}_{s}=\bar{\omega}_{s}\left(\sigma_{s}\right)$, $s=1,2$ is a piecewise uniform mesh with $N_{s}$ intervals on the $x_{s}$-axis. To construct the mesh $\bar{\omega}_{2}\left(\sigma_{2}\right)$, we divide $[0,1]$ in three parts $\left[0, \sigma_{2}\right],\left[\sigma_{2}, 1-\sigma_{2}\right],\left[1-\sigma_{2}, 1\right]$; we take $\sigma_{2}=\min \left[1 / 4, m_{2} \varepsilon \ln N_{2}\right]$. In each part we place a uniform mesh with $N_{2} / 2$

Throughout this paper we denote by $M, M^{(i)}$ (or $m_{i}, m^{(i)}$ ) arbitrary, sufficiently large (small) positive constants independent of $\varepsilon$ and the discretization parameters. 
elements in $\left[\sigma_{2}, 1-\sigma_{2}\right]$ and with $N_{2} / 4$ elements in each subinterval $\left[0, \sigma_{2}\right]$ and $\left[1-\sigma_{2}, 1\right]$. When constructing $\bar{\omega}_{1}\left(\sigma_{1}\right)$, we divide $[0,1]$ in two parts with the transition point $\sigma_{1}=\min \left[1 / 2, m_{1}^{-1} \varepsilon^{2} \ln N_{1}\right]$, where $0<m_{1}<m_{1}^{0}, m_{1}^{0}=$ $\min _{\bar{G}}\left[a_{1}^{-1}(x, t) b_{1}(x, t)\right]$. We place a uniform mesh in $\left[0, \sigma_{1}\right],\left[\sigma_{1}, 1\right]$ using $N_{1} / 2$ mesh elements in each subinterval.

For problem (1) we use the difference scheme [11]

$$
\Lambda_{(2)} z(x, t)=f(x, t), \quad(x, t) \in G_{h}, \quad z(x, t)=\varphi(x, t), \quad(x, t) \in S_{h},
$$

where $\Lambda_{(2)} \equiv \varepsilon^{2} \sum_{s=1,2} a_{s}(x, t) \delta_{\overline{x s} \widehat{x} s}+b_{1}(x, t) \delta_{x 1}-c(x, t)-p(x, t) \delta_{\bar{t}}, \delta_{\bar{t}} z(x, t)$, $\delta_{x 1} z(x, t)$ and $\delta_{\overline{x s} \widehat{s} s} z(x, t)$ are the first and the second differences of $z(x, t)$.

Theorem 1. The solution of finite difference scheme (2), (1) converges $\varepsilon$ uniformly to the solution of (1) with an error bound given by

$$
|u(x, t)-z(x, t)| \leq M\left(N_{1}^{-1} \ln N_{1}+N_{2}^{-2} \ln ^{2} N_{2}+\tau\right), \quad(x, t) \in \bar{G}_{h} .
$$

Remark 1. Let $u \in C^{\beta, \beta / 2}(\bar{G}), \beta=K+2+\alpha, K \geq 0, \alpha>0$. Then the derivatives $\left(\partial^{k_{0}} / \partial t^{k_{0}}\right) u(x, t)$ and the divided differences $\delta_{l \bar{t}} z(x, t)$ satisfy the estimates

$$
\begin{aligned}
\left|\frac{\partial^{k_{0}}}{\partial t^{k_{0}}} u(x, t)\right| & \leq M_{(3)}^{\left(k_{0}\right)}, \quad(x, t) \in \bar{G}, \quad k_{0} \leq K+2 ; \\
\left|\delta_{l \bar{t}} z(x, t)\right| & \leq M_{(4)}^{(l)}, \quad(x, t) \in \bar{G}_{h}, \quad t \geq l \tau, \quad l \leq K+1 .
\end{aligned}
$$

Here $\delta_{l \bar{t}} z(x, t)=\left(\delta_{l-1 \bar{t}} z(x, t)-\delta_{l-1 \bar{t}} z(x, t-\tau)\right) / \tau,(x, t) \in \bar{G}_{h}, t \geq l \tau, l \geq 1$, $\delta_{0 \bar{t}} z(x, t)=z(x, t)$, and $\delta_{l \bar{t}} z(x, t)$ denotes the backward difference of order $l$.

\section{Parallelization of Finite Difference Scheme (2), (1)}

We derive the difference scheme to be solved on $P \geq 1$ parallel processors [8].

1. First we describe a partitioning of the domain $D$

$$
D=\bigcup_{k=1}^{K} D^{k}, \quad D^{k}=(0,1) \times d_{2}^{k},
$$

where $d_{2}^{k}$ are open intervals in $(0,1)$ on the $x_{2}$-axis. Let $G^{k}=D^{k} \times(0, T], k=$ $1, \ldots, K$. We denote the minimal overlap of the sets $D^{k}$ and $D^{[k]}=\bigcup_{i=1, i \neq k}^{K} D^{i}$ by $\delta^{k}$, and by $\delta$ the smallest value of $\delta^{k}$, i.e.,

$$
\begin{gathered}
\min _{k, x^{1}, x^{2}} \rho\left(x^{1}, x^{2}\right)=\delta, \\
x^{1} \in \bar{D}^{k}, \quad x^{2} \in \bar{D}^{[k]}, \quad x^{1}, x^{2} \notin\left\{D^{k} \cap D^{[k]}\right\}, \quad k=1, \ldots, K .
\end{gathered}
$$

In general, the value $\delta$ may depend on the parameter $\varepsilon$.

Let each $D^{k}$ be partitioned into $P$ disjoint (possibly empty) parts

$$
D^{k}=\bigcup_{p=1}^{P} D_{p}^{k}, k=1, \ldots, K, \bar{D}_{i}^{k} \cap \bar{D}_{j}^{k}=\emptyset, i \neq j ; \quad D_{p}^{k}=(0,1) \times d_{2 p}^{k} .
$$


We set $G_{p}^{k}=D_{p}^{k} \times(0, T], \quad p=1, \ldots, P, \quad k=1, \ldots, K$.

We introduce the rectangular grids on each of the sets $\bar{G}^{k}$ and $\bar{G}_{p}^{k}$ :

$$
\bar{G}_{h}^{k}=\bar{G}^{k} \cap \bar{G}_{h(1)}, \quad \bar{G}_{p h}^{k}=\bar{G}_{p}^{k} \cap \bar{G}_{h(1)} .
$$

We define the prizm $G\left(t_{1}\right)$ with the boundary $S\left(t_{1}\right)=\bar{G}\left(t_{1}\right) \backslash G\left(t_{1}\right)$ by

$$
G\left(t_{1}\right)=\left\{(x, t): \quad(x, t) \in G, \quad t_{1}<t \leq t_{1}+\tau\right\}, \quad t_{1}, t_{1}+\tau \in \bar{\omega}_{0} .
$$

Let the discrete function $v\left(x, t ; t_{1}\right)$ be defined at the boundary mesh points $S_{h}\left(t_{1}\right)=S\left(t_{1}\right) \bigcap \bar{G}_{h}, t_{1} \in \bar{\omega}_{0}$. By $\bar{v}\left(x, t ; t_{1}\right)$ we denote the extension of this function to the grid set $\bar{G}_{h}\left(t_{1}\right)=\bar{G}\left(t_{1}\right) \cap \bar{G}_{h}$. The "prizm" $\bar{G}_{h}\left(t_{1}\right)$ consists of only two time levels $\bar{G}_{h}\left(t_{1}\right)=\left\{\bar{D}_{h} \times\left[t=t_{1}\right]\right\} \cup\left\{\bar{D}_{h} \times\left[t=t_{1}+\tau\right]\right\}$.

2. Before to describe the difference scheme designed for parallel implementation on $P$ processors, we assume that $z(x, t)$ is known for $t \leq t^{n}$. Then we solve

$$
\begin{aligned}
& \Lambda_{(2)} z_{p}^{\frac{k}{K}}(x, t)=f(x, t), \quad(x, t) \in G_{p h}^{k}\left(t^{n}\right), \\
& z_{p}^{\frac{k}{K}}(x, t)=\left\{\begin{array}{ll}
\bar{z}\left(x, t ; t^{n}\right), & k=1, \\
z^{\frac{k-1}{K}}(x, t), & k \geq 2
\end{array}\right\}, \quad(x, t) \in S_{p h}^{k}\left(t^{n}\right), \quad p=1, \ldots, P \\
& \text { for }(x, t) \in \bar{G}_{p h}^{k}\left(t^{n}\right), \quad k=1, \ldots, K, \quad t^{n} \in \bar{\omega}_{0}, \quad n \leq N_{0}-1 \text {; }
\end{aligned}
$$

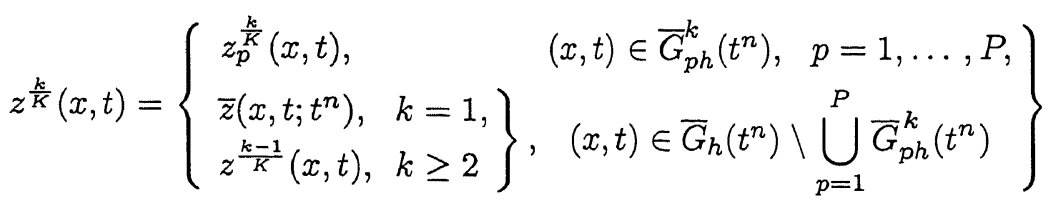

$$
\begin{aligned}
& \text { for }(x, t) \in \bar{G}_{h}\left(t^{n}\right), \quad k=1, \ldots, K, \quad t^{n} \in \bar{\omega}_{0} .
\end{aligned}
$$

We define the function $z_{(5)}(x, t)$ on the prizm $\bar{G}_{h}\left(t^{n}\right)$ by the relation

$$
z_{(5)}(x, t)=z^{\frac{K}{K}}(x, t), \quad(x, t) \in \bar{G}_{h}\left(t^{n}\right), \quad t^{n} \in \bar{\omega}_{0} .
$$

The difference scheme (5) can be written in the operator form

$$
Q_{(5)}\left(z_{(5)}(x, t) ; f(\cdot), \varphi(\cdot), \psi(\cdot)\right)=0, \quad(x, t) \in \bar{G}_{h} .
$$

Here the function $\psi\left(x, t ; t^{n}\right),(x, t) \in G\left(t^{n}\right)$ defines the prolonged function

$$
\bar{z}\left(x, t ; t^{n}\right)=\left\{\begin{array}{ll}
v\left(x, t ; t^{n}\right), & (x, t) \in S\left(t^{n}\right), \\
v\left(x, t^{n} ; t^{n}\right)+\psi\left(x, t ; t^{n}\right), & (x, t) \in G\left(t^{n}\right)
\end{array}\right\}, \quad(x, t) \in \bar{G}\left(t^{n}\right),
$$

where

$$
\left.\begin{array}{c}
v\left(x, t ; t^{n}\right)=\left\{\begin{array}{ccc}
\varphi(x, t), & (x, t) \in S_{h}\left(t^{n}\right), & t^{n}=t^{0}=0, \\
\varphi(x, t), & (x, t) \in S_{h}\left(t^{n}\right) \cap S_{h}, & t \geq t^{n}, \\
z(x, t), & (x, t) \in S_{h}\left(t^{n}\right) \backslash S_{h}, & t=t^{n}
\end{array}\right\}, t^{n}>0
\end{array}\right\},
$$


In the specific problem (5) we take $\psi\left(x, t ; t^{n}\right) \equiv 0$.

Note that the intermediate problems in the discrete DD method (5), (4) are solved on the subsets $\bar{D}_{p h}^{k}=\bar{D}_{p(3)}^{k} \cap \bar{D}_{h}$ independently of each other ("in parallel") for all $p=1, \ldots, P$.

Let the following condition be satisfied

$$
\delta=\delta_{(2)}(\varepsilon)>0, \quad \varepsilon \in(0,1], \quad \inf _{\varepsilon \in(0,1]}\left[\varepsilon^{-1} \delta_{(2)}(\varepsilon)\right]>0 .
$$

A technique similar to the one exposed in $[6,7]$ gives us the error estimate

$$
\left|u(x, t)-z_{(5)}(x, t)\right| \leq M\left(N_{1}^{-1} \ln N_{1}+N_{2}^{-2} \ln ^{2} N_{2}+N_{0}^{-1}\right), \quad(x, t) \in \bar{G}_{h} .
$$

Theorem 2. Under condition (6) and for $N, N_{0} \rightarrow \infty$, the solution of the difference scheme (5), (4) converges to the solution of (1) $\varepsilon$-uniformly. The estimate (7) holds for the solution of this difference scheme.

\section{Improved Time-Accuracy. Parallel Scheme}

1. Constructing the defect-correction difference scheme on $\bar{G}_{h}$, we rewrite the finite difference scheme (2) as in [7]:

$$
\Lambda_{(2)} z^{(1)}(x, t)=f(x, t), \quad(x, t) \in G_{h}, \quad z^{(1)}(x, t)=\varphi(x, t), \quad(x, t) \in S_{h},
$$

where $z^{(1)}(x, t)$ is the uncorrected solution. To find the corrected solution $z^{(2)}(x, t)$, we solve the problem

$$
\begin{gathered}
\Lambda_{(2)} z^{(2)}(x, t)=f(x, t)+\left\{\begin{array}{l}
2^{-1} p(x, t) \tau \frac{\partial^{2}}{\partial t^{2}} u(x, 0), \quad t=\tau, \\
2^{-1} p(x, t) \tau \delta_{2 \bar{t}} z^{(1)}(x, t), t \geq 2 \tau
\end{array}\right\},(x, t) \in G_{h}, \\
z^{(2)}(x, t)=\varphi(x, t), \quad(x, t) \in S_{h} .
\end{gathered}
$$

Here the derivative $\left(\partial^{2} / \partial t^{2}\right) u(x, 0)$ is obtained from equation (1a).

In the remainder of this section we consider a homogeneous initial condition

$$
\varphi(x, 0)=0, \quad x \in \bar{D} \text {. }
$$

Under this condition, for the solution of problem (2), (1) we have

$$
\left|u(x, t)-z^{(2)}(x, t)\right| \leq M\left[N_{1}^{-1} \ln N_{1}+N_{2}^{-2} \ln ^{2} N_{2}+\tau^{2}\right], \quad(x, t) \in \bar{G}_{h} .
$$

Proceeding in a similar way, one can construct difference schemes with a higher order of time-accuracy $\mathcal{O}\left(\tau^{l}\right), \quad l>2$ (see $[7,8]$ for $l=3$ ).

2. Let us consider a parallel version for the defect correction scheme. In the operator form the above difference scheme is written as follows

$$
\begin{array}{ll}
Q_{(5)}\left(z^{(1)}(x, t) ; f^{(1)}(\cdot), \varphi(\cdot), \psi^{(1)}(\cdot)\right)=0, & (x, t) \in \bar{G}_{h}, \\
Q_{(5)}\left(z^{(2)}(x, t) ; f^{(2)}(\cdot), \varphi(\cdot), \psi^{(2)}(\cdot)\right)=0, & (x, t) \in \bar{G}_{h},
\end{array}
$$


where

$$
\begin{aligned}
& f^{(1)}(x, t)=f(x, t), \quad f^{(2)}(x, t)=f^{(2)}\left(x, t ; z^{(1)}(\cdot)\right)=f(x, t)+ \\
&+\left\{\begin{array}{l}
2^{-1} p(x, t) \tau\left(\partial^{2} / \partial t^{2}\right) u(x, 0), t=\tau, \\
2^{-1} p(x, t) \tau \delta_{2 \bar{t}} z^{(1)}(x, t), \quad t \geq 2 \tau
\end{array}\right\}, \quad(x, t) \in G_{h}^{k}, \\
& \psi^{(1)}\left(x, t ; t^{n}\right) \equiv 0, \quad \psi^{(2)}\left(x, t ; t^{n}\right)=\psi^{(2)}\left(x, t ; t^{n}, z^{(1)}(\cdot)\right)= \\
&=z^{(1)}\left(x, t^{n+1}\right)-z^{(1)}\left(x, t^{n}\right), \quad(x, t) \in G_{h}\left(t^{n}\right), \quad t=t^{n+1} .
\end{aligned}
$$

It is easy to see that $z^{(1)}(x, t) \equiv z_{(5 ; 4)}(x, t)$.

Following the arguments from $[6,7,9]$ we obtain the main convergence result.

Theorem 3. Let condition (3) hold. Then, under condition (6), the solution of the difference scheme (5), (4) converges, as $N, N_{0} \rightarrow \infty$, to the solution of the boundary value problem (1) E-uniformly. For the discrete solution the estimate (4) holds.

\section{Acceleration of Computations by the Parallel Scheme}

To solve the problem (1), we use scheme (2), (1) with improved time-accuracy as the base scheme. One can also use the parallel variant of scheme (5), (4). We say that the use of parallel computations leads to the real acceleration of the solution process if such a scheme with $P>1$ parallel processors can be found for which the computation time turns out to be smaller and the accuracy of the approximate solution is not lower than those for the base scheme.

We shall consider the difference scheme for $P$ parallel solvers on the meshes

$$
\bar{G}_{p h}^{k P}=\bar{G}_{p}^{k} \cap \bar{G}_{h}^{P}, \quad \bar{G}_{h}^{P}=\bar{D}_{h} \times \bar{\omega}_{0}^{P},
$$

where $\bar{D}_{h}=\bar{D}_{h(1)}, \bar{\omega}_{0}^{P}$ is a uniform mesh on $[0, T]$ with the number of nodes $N_{0}^{P}+1$ and the mesh step $\tau^{P}$; generally speaking, $\bar{\omega}_{0(1)}^{P} \neq \bar{\omega}_{0(1)}$.

1. We now describe the decomposition of the set $\bar{D}$ which can ensure the acceleration of the solution process.

Let the domain $D$ consist of $J$ non-overlapping rectangles

$$
D^{<j>}, \quad j=1, \ldots, J,
$$

where $D^{<i>} \cap D^{<j>}=\emptyset$ for $i \neq j, \quad \bar{D}=\bigcup_{j=1}^{J} \bar{D}^{<j>} ; J \leq M$. On each of the sets $\bar{G}^{<j>}=\bar{D}^{<j>} \times[0, T]$, the mesh $\bar{G}_{h}$ with the given distribution of its nodes generates the meshes $\bar{G}_{h}^{<j>}=\bar{G}^{<j>} \cap \bar{G}_{h}, j=1, \ldots, J, \bar{G}_{h}=\bar{G}_{h(1)}^{P}$. For each of the sets $D^{<j>}$ we construct the rectangle $D^{j}$ containing $D^{<j>}$ together with some neighborhood. This set $\bar{D}^{j}$ satisfies the three conditions: 
(a) $\bar{D}^{j}$ contains the set of the points distant from $\bar{D}^{<j>}$ on the distance which is not smaller than $\delta_{0}$, where

$$
\delta_{0}=m_{(2)}^{(1)} \varepsilon \text { with some fixed } m_{(2)}^{(1)} ;
$$

(b) the sides of the set $\bar{G}^{j}=\bar{D}^{j} \times[0, T]$ pass through the nodes of the mesh $\bar{G}_{h}$;

(c) the number of nodes in each of the meshes $\bar{D}_{h}^{j}=\bar{D}^{j} \cap \bar{D}_{h}$ is the same and it does not depend on the number $j$.

Let the work time of the processors, assigned for resolving the discrete problem on the level $t=t^{1}$ of the mesh set $\bar{D}_{h}^{0}$ from $\bar{D}_{h(1)}$, be defined by the value $\mu\left(\bar{D}_{h}^{0}\right)$, that is the number of nodes in the set $\bar{D}_{h}^{0}$.

The sets

$$
G^{j}=D^{j} \times(0, T], \quad j=1, \ldots, J,
$$

form the preliminary covering of the set $G$, that is, $G=\bigcup_{j=1}^{J} G^{j}$. Assume

$$
\mu\left(\bar{D}_{h}^{j}\right)=\mu^{0}, \quad \mu\left(\bar{D}_{h}^{j}\right)=\left(1+m_{(2)}^{(2)}\right) \mu\left(\bar{D}_{h}^{<j>}\right), \quad j=1, \ldots, J .
$$

The sets (2c) are used for the construction of the special DD scheme (5), (1) with $P$ processors. For this, we construct the sets

$$
G^{\{k\}}, \quad k=1, \ldots, K
$$

which cover the set $G$, where the value $K=K(P)$ is chosen from the condition $K P=J$. The each of the sets $G^{\{k\}}$ is multiply connected (for $P>1$ ) and formed by the union of the $P$ non-overlapping domains from (2c). Thus, for the subsets $G_{p}^{k}$ which form the sets from (3a), the following condition holds:

$$
G_{p}^{k} \subset\left\{G^{j}, j=1, \ldots, J\right\}_{(2 \mathrm{c})}, \quad k=1, \ldots, K, \quad p=1, \ldots, P,
$$

where $\mu\left(\bar{D}_{p h}^{k}\right)=\mu^{0}, G^{\{k\}}=\bigcup_{p=1}^{P} G_{p}^{k}$. With such decomposition the processors are loaded more effectively.

2. By definition, we denote the work time, which is required to solve problems (2), (1) and (5), (4) respectively, by

$$
\vartheta=\vartheta\left(N_{0}\right) \equiv N_{0} \mu\left(\bar{D}_{h}\right), \quad \vartheta^{P}=\vartheta^{P}\left(N_{0}^{P}, P\right) \equiv N_{0}^{P} \sum_{k=1}^{K} \max _{p} \mu\left(\bar{D}_{p h}^{k}\right) .
$$

Then the rate of acceleraton for our computations is defined by

$$
C=C\left(N_{0}, N_{0}^{P}, P\right)=\vartheta\left(\vartheta^{P}\right)^{-1} \equiv N_{0}\left(N_{0}^{P}\right)^{-1} \mu\left(\bar{D}_{h}\right)\left\{\sum_{k=1}^{K(P)} \max _{p} \mu\left(\bar{D}_{p h}^{k}\right)\right\}^{-1} .
$$

3. We now give the conditions ensuring the acceleration of the solution process based on parallelization of scheme (2), (1). Here we assume that the derivative 
$\left(\partial^{3} / \partial t^{3}\right) u(x, t)$ on the set $\bar{G}$ is not too small. Precisely, let the following condition hold

$$
\left|\frac{\partial^{3}}{\partial t^{3}} u(x, t)\right| \geq m^{(3)}, \quad(x, t) \in \bar{G}^{*}
$$

on some set $\bar{G}^{*}=\left\{(x, t): x_{s}^{* 1} \leq x_{s} \leq x_{s}^{* 2}, s=1,2, t^{* 1} \leq t \leq t^{* 2}\right\}, \bar{G}^{*} \subseteq \bar{G}$.

In the case when the number $P$ of processors is sufficiently large, i.e.,

$$
P>M\left(1+m_{(2)}^{(2)}\right)\left(m_{(4)}^{(3)}\right)^{-1}\left[M_{(3)}^{(3)}+M_{(3)}^{(4)}+M_{(3)}^{(5)}\right] \equiv P^{*},
$$

the acceleration can be really attained for the numerical solution of the boundary value problem. In fact, the acceleration is achieved under the condition

$$
N_{0}^{P}=\left(1+m_{(2)}^{(2)}\right)^{-1} N_{0} P^{*} .
$$

The value of $C$, which characterizes the attained rate of acceleration, is defined by

$$
C=P\left(P^{*}\right)^{-1}, \quad P^{*}=P_{(5)}^{*} .
$$

Theorem 4. Let conditions (3), (4), (4) hold for the solutions of the boundary value problem (1) and scheme (2), (1). Then, in the class of difference schemes (5), (1) for $P$ parallel processors, $\varepsilon$-uniform acceleration of solving problem (1), as compared to the base scheme (2), (1), can be achieved in general; in particular, for the decomposition (3), (1) the acceleration is achievable under condition (5). Moreover, for scheme (5), (3), (1) the acceleration is attained under conditions (5), (6), and the rate $C$ of acceleration is defined by (7).

\section{References}

1. Farrell, P. A., Hemker, P. W., Shishkin, G. I.: Discrete approximations for singularly perturbed boundary value problems with parabolic layers. $I$, J. Comput. Math. 14(1) (1996) 71-97; II, J. Comput. Math. 14(2) (1996) 183-194; III, J. Comput. Math. 14(3) (1996) 273-290

2. Farrell, P. A., Miller, J. J. H., O'Riordan, E., Shishkin, G. I.: A uniformly convergent finite difference scheme for a singularly perturbed semilinear equation. SIAM J. Numer. Anal. 33 (1996) 1135-1149

3. Shishkin, G. I.: Grid Approximations of Singularly Perturbed Elliptic and Parabolic Equations (in Russian). Ural Branch of Russian Acad. Sci., Ekaterinburg (1992)

4. Miller, J. J. H., O'Riordan, E., Shishkin G. I.: Fitted Numerical Methods for Singular Perturbation Problems. World Scientific, Singapore (1996)

5. Roos, H.-G., Stynes, M., Tobiska, L.: Numerical Methods for Singularly Perturbed Differential Equations. Springer-Verlag, Berlin (1996)

6. Hemker, P. W., Shishkin, G. I., Shishkina, L. P.: The use of defect correction for the solution of parabolic singular perturbation problems. ZAMM 77 (1997) 59-74 
7. Hemker, P. W., Shishkin, G. I., Shishkina, L. P.: $\varepsilon$-uniform schemes with highorder time-accuracy for parabolic singular perturbation problems. IMA J. Numer. Anal. 20 (2000) 99-121

8. Hemker, P. W., Shishkin, G. I., Shishkina, L. P.: Distributing the numerical solution of parabolic singularly perturbed problems with defect-correction over independent processes. Siberian J. Numer. Mathematics, Novosibirsk (to appear)

9. Shishkin, G. I.: Acceleration of the process of the numerical solution to singularly perturbed boundary value problems for parabolic equations on the basis of parallel computations. Russ. J. Numer. Anal. Math. Modelling 12 (1997) 271-291

10. Shishkin, G. I.: Grid approximation of singularly perturbed elliptic equation in domain with characteristic bounds. Sov. J. Numer. Anal. Math. Modelling 5 (1990) 327-343

11. Samarskii, A. A.: Theory of Difference Schemes (in Russian). Nauka, Moscow (1989) 